\section{Target for ecosystem repair is impractical}

The Convention on Biological Diversity (CBD) agreed in 2010 to restore "at least 15 per cent of degraded ecosystems" by 2020 (www.cbd.int/sp/targets). In Finland's experience, this target is unrealistic.

Ecosystem destruction is measured according to the expanse and degree of degradation. Consequently, under the CBD agreement, damage to an ecosystem's condition needs to be reduced by $15 \%$ over an entire area of degraded landscape or, for example, by $33 \%$ in a randomly selected $45 \%$ of that area to attain the same reduction.

There are considerable challenges in achieving this reduction: heavy restoration measures must be completed across large areas and in a short time, while compensating for ongoing degradation elsewhere.

Finland's forests cover roughly 15 million hectares, $95 \%$ of which have been degraded by forestry operations. To meet the CBD target, the country would need to reduce degradation by $33 \%$ in more than 100,000 hectares each month until 2020.

It has already taken Finland almost 30 years to restore some 30,000 hectares of forest (see Table 2.5 at go.nature.com/pajic9) - one-thousandth of the CBD's monthly requirement.

There is a danger that parties to the $\mathrm{CBD}$ will ignore the target because of its sheer impracticality. Janne S. Kotiaho ${ }^{\star}$ University of Jyväskylä, Finland. janne.kotiaho@jyu.fi ${ }^{\star}$ On behalf of 7 correspondents (see go.nature.com/dcyoft for full list).

\section{Sports doping vastly underestimated}

Roger Pielke Jr suggests that doping prevalence can be estimated by drug testing of athletes (Nature 517, 529; 2015). I contend that this method is flawed: as the autobiographies of some athletes attest, regular dopers have a track record of avoiding testing positive.

To estimate doping's true prevalence, two procedures that circumvent inherent weaknesses in simple counts of positive test results are useful.

First, Bayesian inference methods can be used to compare two distributions of biological parameters affected by doping: one distribution among sampled athletes and the other in a suitable reference population, allowing the number of manipulated samples to be estimated (P.-E. Sottas et al. Clin. Chem. 57, 762-769; 2011). A major advantage of this population-level analysis is that it can recognize abnormalities even when dopers' values remain within the 'normal' range.

Second, specially tailored questionnaires allow athletes to give honest answers to doping questions under cover of anonymity (W. Pitsch et al. Eur. J. Sport. Soc. 4, 89-102; 2007). The questionnaires are based on the randomized response technique and are used by social scientists to study illegal and deviant behaviours.

A combination of these approaches estimates that 14-39\% of elite athletes have intentionally doped (see O. de Hon et al. Sports Med. 45, 57-69; 2015). This contrasts markedly with the $2 \%$ of samples designated as suspect in the World Anti-Doping Agency's published statistics.

Simon Evans Uppsala University, Uppsala, Sweden.

simon.evans@ebc.uu.se

\section{A marine biologist's remarkable legacy}

We write to dispel some myths in characterizations of the marine biologist Ed Ricketts, who died in 1948 (see, for example, A. Hirsh Nature 516, 326-328; 2014).

Far from working in isolation, Ricketts interacted and corresponded with Torsten
Gislén, George MacGinitie and Willis Hewatt, who all studied intertidal creatures. He did the same with scientists at the Smithsonian Institution in Washington DC and those at US and foreign universities. Never having taken a university degree, he was eager to tap into their expertise and to contribute to the international body of knowledge.

Hirsh's observation that Ricketts' book, Between Pacific Tides, was revolutionary because "it categorizes animals according to habitat, not phylum or family" is not the whole story. Ricketts' years of data from tidepool observations and collections resulted in a complex cardindexing and cross-referencing system that allowed him to build conclusions about species and their habitats and animal communities. As a result, his ecological data sets are still among the most robust ever to have been assembled.

Steven Albert, Mary Albert Pacific Grove, California, USA. Don Kohrs Hopkins Marine Station of Stanford University, Pacific Grove, California, USA. alberts1234@comcast.net

\section{German initiative opens up animal data}

Reliable and transparent data are essential to discussions of suffering in animal experimentation. A new initiative in Germany provides user-friendly public information about authorized animalresearch projects.

The European directive to protect laboratory animals (2010/63/EU) requires researchers to provide an anonymous, non-technical summary of a proposed project, stating its purpose and potential benefits. The summaries also detail the number and types of animal to be used, the predicted harm to the animals and the evidence of compliance with the ' $3 R$ ' principles (see go.nature. com/jg7n6v). The severity of animal suffering and the likely human benefits are central to the approval process.

Germany's Federal Institute for Risk Assessment (BfR) has created a freely accessible, searchable website of these summaries to provide the public with clear insight into animal experiments (see www. animaltestinfo.de). Summaries are available to all EU member states, which could all set up similar open-access databases.

The summaries provide a unique channel for scientists to communicate their work to the public. They are a milestone in attempts to safeguard transparency in animal research, which is particularly controversial in the case of nonhuman primates.

Gilbert Schönfelder ${ }^{\star} B f R$, Berlin; and Charité - University Medicine Berlin, Germany. gilbert.schoenfelder@bfr.bund.de ${ }^{*}$ On behalf of 4 correspondents (see go.nature com/ebzmxo for full list).

\section{Glass-blowing's Nobel moment}

One of the most striking contributions of glass-blowing to research (see Nature 517, $542-546$; 2015) is the creation of 11,146 hemispherical photomultipliers in the neutrino detector used by Nobel laureate Masatoshi Koshiba. Their 50-centimetre diameters are a phenomenal achievement that called for great skill, strength and exceptional lung capacity.

Koshiba was awarded the Nobel Prize in Physics in 2002 for the detection of cosmic neutrinos at the Kamioka Observatory in Hida, Japan. He acknowledged the glass-blowers of the Hamamatsu Photonics firm in his Nobel lecture, saying that the 50-cm-diameter photomultipliers "were the essential ingredient of the Kamioka experiments". Min-Liang Wong National Chung-Hsing University, Taichung, Taiwan. mlwong@dragon.nchu.edu.tw 